\title{
Getting a kick out of numerical relativity.
}

\author{
John G. Baker, Joan Centrella, Dae-Il Choi ${ }^{1}$, Michael Koppitz, James R. van Meter, \\ M. Coleman Miller ${ }^{2}$ \\ Laboratory for Gravitational Astrophysics, NASA Goddard Space Flight Center, Greenbelt, \\ Maryland 20771
}

\begin{abstract}
Recent developments in numerical relativity have made it possible to follow reliably the coalescence of two black holes from near the innermost stable circular orbit to final ringdown. This opens up a wide variety of exciting astrophysical applications of these simulations. Chief among these is the net kick received when two unequal mass or spinning black holes merge. The magnitude of this kick has bearing on the production and growth of supermassive black holes during the epoch of structure formation, and on the retention of black holes in stellar clusters. Here we report the first accurate numerical calculation of this kick, for two nonspinning black holes in a 1.5:1 mass ratio, which is expected based on analytic considerations to give a significant fraction of the maximum possible recoil. Our estimated kick is $105 \mathrm{~km} \mathrm{~s}^{-1}$ with an error of less than $10 \%$. This is intermediate between the estimates from two recent post-Newtonian analyses and suggests that at redshifts $z \gtrsim 10$, halos with masses $\lesssim 10^{9} M_{\odot}$ will have difficulty retaining coalesced black holes after major mergers.
\end{abstract}

Subject headings: black hole physics — gravitational waves — relativity — cosmology: theory

\section{Introduction}

When two black holes merge, the gravitational waves they produce will carry away net linear momentum, barring conditions of special symmetry (e.g., two equal-mass nonspinning black holes receive no kick). The magnitude of the resulting recoil is important in a variety

\footnotetext{
${ }^{1}$ Universities Space Research Association, 10211 Wincopin Circle, Suite 500, Columbia, MD 21044

${ }^{2}$ University of Maryland, Department of Astronomy, College Park, MD 20742-2421
} 
of astrophysical contexts, including the cosmological evolution of supermassive black holes (Merritt et al. 2004; Boylan-Kolchin, Ma, \& Quataert 2004; Haiman 2004; Madau \& Quataert 2004; Yoo \& Miralda-Escudé 2004; Volonteri \& Perna 2005; Libeskind et al. 2005; Micic, Abel, \& Sigurdsson 2005) and the growth and retention of intermediate-mass black holes in dense stellar clusters (Taniguchi et al. 2000; Miller \& Hamilton 2002a,b; Mouri \& Taniguchi 2002a,b; Miller \& Colbert 2004; Gültekin, Miller, \& Hamilton 2004, 2006; O'Leary et al. 2006). There is therefore a long history of analytical estimates of this recoil (Peres 1962; Bekenstein 1973; Fitchett 1983; Fitchett \& Detweiler 1984; Redmount \& Rees 1989; Wiseman 1992; Favata, Hughes, \& Holz 2004; Blanchet, Qusailah, \& Will 2005; Damour \& Gopakumar 2006). However, it has been shown that almost all of the recoil occurs in the strong gravity regime, inside the innermost stable circular orbit (ISCO). This is precisely where analytical treatments are least reliable. An accurate estimate of the recoil kick therefore requires full numerical simulations of the final phase of the inspiral, merger, and ringdown of the coalescence of two black holes.

Until recently, numerical simulations were not stable and accurate enough for such estimates. This situation has changed dramatically in the past year, with several groups developing codes that have allowed the evolution of binary black hole spacetimes from close to the ISCO through merger and ringdown. Most of these codes utilize techniques which allow the black holes, with their inherent singularities, to move successfully through the computational domain. One approach (Pretorius 2005, 2006) is to excise the singular region in the (physically inaccessible) interior of the black hole. In contrast, our method allows the singular region to be approximately represented in the computational domain (Baker et al. 2005, 2006; Campanelli et al. 2005b). These new techniques have led to dramatically more effective numerical simulations of binary black hole systems, recently allowing us to determinate accurate waveforms for the final orbits and merger of equal mass systems (Baker et al. 2006), which we generalize here for nonequal masses. While some preliminary numerical calculations of recoil from mergers of non-spinning black holes have been reported (Campanelli 2005a; Herrman, Shoemaker, \& Laguna 2006), the initial separations have been too small and the resolutions too coarse for reliable and precise numbers.

Here we report the first precise fully numerical estimates of the kick received from the merger of two nonspinning black holes. We choose a 1.5:1 mass ratio because it is close to the analytically estimated optimal mass ratio for maximum kick, but is also close enough to equal mass that resolution issues are not serious impediments to the numerical evolution. In $\S 2$ we describe our numerical method and results, including convergence tests. We discuss the astrophysical implications of these results in $\S 3$. 


\section{Numerical Simulations}

Reliable simulations of binary black hole mergers requires the specification of constraintsatisfying initial field data followed by stable evolution of the Einstein equations. The evolution variables hold information about gravitational fields in the form of tensor fields representing the curvature of a vacuum spacetime. Einstein's equations, together with a crucial specification of gauge (i.e., coordinate) conditions, then govern the evolution of these fields in time.

We utilize the "puncture" approach (Brandt \& Brügmann 1997) to specify constraintsatisfying initial field configurations for black holes on an approximately circular inspiral trajectory within an orbit or two of merger. Specifically, we consider two cases of initial configurations, with the black holes at initial coordinate separations of $d_{\text {init }}=4.1 M_{0}$ and $d_{\text {init }}=6.2 M_{0}$, where $M_{0}$ is the total initial gravitational mass of the system, and we use units where Newton's gravitational constant $G$ and the speed of light $c$ are set to unity so that all quantities can be represented in terms of their mass-scaling. These data begin our simulations as the system approaches the ISCO, allowing respectively about 1 or 2.5 orbits before the formation of a common horizon representing the final black hole.

Hahndol (Imbiriba et al. 2004), using a second-order iterative Crank-Nicholson scheme, on an adaptive mesh refinement structure implemented via PARAMESH (MacNeice et al. 2000). The punctures are allowed to move freely through the grid and are evolved according to a variant of the BSSN formulation of Einstein's equations and gauge conditions as described in Baker et al. (2006).

We interpret our numerical results by studying the gravitational radiation generated by the merger which carries gauge-invariant information away from the strong-field region. In our simulations, radiation is represented by a component of the space-time Weyl curvature tensor, $\psi_{4}$. In terms of $\psi_{4}$, the net momentum radiated by the system can be expressed as follows:

$$
\frac{d P_{i}}{d t}=\lim _{r \rightarrow \infty}\left\{\frac{r^{2}}{4 \pi} \int d \Omega \frac{x_{i}}{r}\left|\int_{-\infty}^{t} \psi_{4}\right|^{2}\right\}
$$

We compute $\psi_{4}$ numerically, and extract it from the simulation data on a sphere of radius $r=40 M_{0}$, which we have found is sufficiently large enough to give accurate results.

Our main numerical results are given in Figure 1, which shows the net speed of the center of mass as a function of time for several runs. To compare runs at different initial coordinate separations we have shifted the time axis so that the kick speed peaks at $t=0$. Note that for the $d_{\text {init }}=4.1 M_{0}$ case we show the results of two runs with different numerical resolutions, 
which give kick histories and final kicks that are almost indistinguishable, differing by less than $1 \%$. This provides an indication of the accuracy of the simulations. Note also that the run with separation $d_{\text {init }}=6.2 M_{0}$ gives a final kick just $10 \%$ less than the closer runs, suggesting that the sensitivity of the result to the choice of initial configuration has only a small effect, which we expect to be less important for the case of larger initial separation.

The inspiral from infinity to our starting point would, in reality, already have imparted a small amount of momentum to the center of mass by the beginning of our runs. Without a specific expression for this we have simply assumed vanishing initial momentum, $P_{\text {init }}=0$, in integrating (1) to get most of kick histories shown in Figure 1. To explore the effects of this in a simple way, we include an additional (thick solid) curve in Figure 1, which was generated from the same data of our larger separation run, but assuming a nonvanishing initial momentum vector in the kick integral, with the value chosen to smooth out the evolution of the kick speed. The initial value used for this curve had a magnitude of a few $\mathrm{km} \mathrm{s}^{-1}$, comparable to the value suggested by post-Newtonian analyses (e.g., Blanchet, Qusailah, \& Will 2005). The modified curve shows a much smoother approach to the final kick, though the final value is almost exactly the same. Though this will need to be confirmed by runs with larger initial separation, it does suggest that, as expected analytically, the net kick grows smoothly and monotonically until the merger.

Recently, two groups have made refined analytic estimates of the kick from merger of nonspinning black holes, both with precise answers but differing in magnitude by a factor of three. Blanchet, Qusailah, \& Will (2005) predict a speed of $155 \pm 25 \mathrm{~km} \mathrm{~s}^{-1}$ for a 1.5:1 mass ratio, whereas Damour \& Gopakumar (2006) predict $48 \mathrm{~km} \mathrm{~s}^{-1}$. Our result is between these estimates but inconsistent with either one. An initial numerical estimate for 1.04:1 and 1.18:1 mass ratios was made by Herrman, Shoemaker, \& Laguna (2006) (albeit at much lower resolution and starting much closer than our simulations), and they find kick speeds of $9 \mathrm{~km} \mathrm{~s}^{-1}$ and $33 \mathrm{~km} \mathrm{~s}^{-1}$, respectively. One can compare results at different mass with reference to the Fitchett (1983) fitting formula kick $\propto q^{2}(q-1) /(1+q)^{5}=(\mu / m)^{2} \mathrm{~d} m / m$, where $q=m_{1} / m_{2}>1$ is the mass ratio, $\mu / m=m_{1} m_{2} /\left(m_{1}+m_{2}\right)^{2}$ is the symmetric mass ratio and $\mathrm{d} m / m=\left(m_{1}-m_{2}\right) /\left(m_{1}+m_{2}\right)$ is the fractional mass difference. Although originally derived in the context of a leading order post-Newtonian approximation, this formula is suggested to be a good approximation by perturbative Schwarzschild calculations (Fitchett \& Detweiler 1984), and it also closely agrees with more recent post-Newtonian results (Blanchet, Qusailah, \& Will 2005; Damour \& Gopakumar 2006). By Fitchett's formula, the Herrman, Shoemaker, \& Laguna (2006) results would imply $\sim 80 \mathrm{~km} \mathrm{~s}^{-1}$ for a mass ratio of 1.5:1, closer to our value than the analytic estimates but still below ours. However, the convergence of our results in the numerical and physical domains suggests that our kick is correct to $\lesssim 10 \%$ and can therefore be used to test strong-field extrapolations of post-Newtonian analyses as 
well as to investigate astrophysical consequences.

\section{Discussion and Conclusions}

Our estimate of $105 \pm 10 \mathrm{~km} \mathrm{~s}^{-1}$ for the kick received in a 1.5:1 merger of nonspinning black holes has important implications for the assembly of supermassive black holes in the early universe (see also Merritt et al. 2004). This is because as dark matter halos merge in the process of hierarchical structure assembly, their central black holes are also presumed to merge if the halo mass ratio is not too extreme (otherwise dynamical friction on the halos is likely to be inefficient; see Taffoni et al. 2003). If black hole masses are linked to the mass of their host halos, it is therefore expected that in the early universe, black hole mergers are likely to involve comparable-mass objects. If the resulting kick exceeds the escape speed of the merged dark matter halo, the halo is left without a black hole. This could, therefore, have a significant impact on the number of black hole mergers in the early universe. In addition, if mergers between comparable-mass halos are common at redshifts $z \gtrsim 10$, these ejections might reduce substantially the fraction of halos that host black holes.

A full appraisal of the consequences of recoil will require detailed numerical studies of the effects of black hole spin and mass ratio, which will be our subject in future papers. We can make initial guesses by focusing on nonspinning black holes and by adopting as before the Fitchett (1983) formula for the dependence of kick speed on mass ratio. Setting $v_{\text {kick }}(q=1.5)=105 \mathrm{~km} \mathrm{~s}^{-1}$ fixes the curve.

To estimate the escape speed from a dark matter halo, we follow the treatment of Merritt et al. (2004). The escape speed from a halo of virial mass $M_{\mathrm{vir}}$ is $V_{\mathrm{esc}}^{2}=2 c g(c) M_{\mathrm{vir}} / R_{\mathrm{vir}}$, where as before we set $G=1$. Here $c$ is the concentration parameter for the halo, $g(c)=$ $[\ln (1+c)-c /(1+c)]^{-1}$, and we follow Navarro, Frenk, \& White $(1997)$ in defining $R_{\text {vir }}$ as the radius inside which the average density is 200 times critical (and thus ignoring the very weak dependence on cosmology): $R_{\text {vir }}=\left[M_{\text {vir }} /\left(200 \times 4 \pi \rho_{\text {crit }} / 3\right)\right]^{1 / 3}$, where $\rho_{\text {crit }}=3 H^{2} /(8 \pi) \approx$ $10^{-29} \mathrm{~g} \mathrm{~cm}^{-3}(1+z)^{3}$. Bullock et al. (2001) find that the concentration parameter scales as $c=9\left[M_{\mathrm{vir}} /\left(2 \times 10^{13} M_{\odot}\right)\right]^{-0.13}(1+z)^{-1}$. The resulting escape speed is

$$
V_{\text {esc }} \approx 220 \mathrm{~km} \mathrm{~s}^{-1}[g(c)]^{1 / 2}\left(M_{\mathrm{vir}} / 10^{10} M_{\odot}\right)^{0.27} \text {. }
$$

In Figure 2 we plot the minimum halo mass as a function of redshift such that $V_{\text {esc }}>v_{\text {kick }}$ for the listed mass ratios of 1.5:1, 3:1, 5:1, and 10:1. Note that the low power of $M_{\text {vir }}$ in the escape speed means that the minimum halo mass depends sensitively on the kick speed. For example, if the kick speed at a 1.5:1 mass ratio were $200 \mathrm{~km} \mathrm{~s}^{-1}$ instead of $105 \mathrm{~km} \mathrm{~s}^{-1}$, the threshold halo mass at $z=10$ for this mass ratio would jump from $4 \times 10^{8} M_{\odot}$ to $3 \times 10^{9} M_{\odot}$. 
This would in turn reduce the number density of halos of at least the required mass by a factor 20 (see Mo \& White 2002 for a pedagogical discussion of how to estimate halo number densities). This points out the importance of estimating kick speeds accurately.

As discussed by Merritt et al. (2004), kicks are also important in the current-day universe for low-mass concentrations of stars. Their Figure 2 is a useful summary of escape speeds from the centers of galaxies and globular clusters. From their figure, we see that comparablemass mergers with kick speeds $\sim 100 \mathrm{~km} \mathrm{~s}^{-1}$ will cause ejection from globulars and some dwarf galaxies, but that more massive galaxies will retain the remnant. Using the Fitchett (1983) scaling with mass ratio, we find that black holes that are $\gtrsim 10$ times more massive than their companions are not ejected from globular clusters with escape speeds of a few tens of $\mathrm{km} \mathrm{s}^{-1}$. Coincidentally, this is approximately the same mass ratio required to protect a massive black hole from cluster ejection from three-body interactions (Gültekin, Miller, \& Hamilton 2004). Therefore, if an intermediate-mass black hole of $>100-200 M_{\odot}$ is formed in a cluster, it can stay and potentially grow through future interactions. Stellar-mass binary black holes $\left(M<50 M_{\odot}\right)$ will be ejected from such clusters prior to merger by Newtonian three-body interactions (Kulkarni, Hut, \& McMillan 1993; Sigurdsson \& Hernquist 1993), hence recoil is not so important for low-mass black holes in this context. However, mergers of black hole binaries in low-density galactic disks could produce a population of high-speed coalesced black holes.

In conclusion, we have presented the first reliable and precise fully numerical estimate of the gravitational recoil produced by the merger of two unequal mass nonspinning black holes. Our value of $105 \mathrm{~km} \mathrm{~s}^{-1}$ for a $1.5: 1$ mass ratio is accurate to within $\lesssim 10 \%$, as determined by our runs at different resolutions and different initial separations. This speed is intermediate between the recent analytic estimates of Blanchet, Qusailah, \& Will (2005), who suggest $155 \pm 25 \mathrm{~km} \mathrm{~s}^{-1}$ for this mass ratio, and Damour \& Gopakumar (2006), whose formulae would imply $48 \mathrm{~km} \mathrm{~s}^{-1}$. Our results are thus an important first step in the evaluation of the astrophysical consequences of gravitational radiation recoil in dense stellar clusters and the early universe.

We gratefully acknowledge supercomputing support from the Commodity Cluster Computing Project (NASA-GSFC), the NASA Center for Computational Sciences (NASA-GSFC) and Project Columbia (NASA Advanced Supercomputing Division, NASA-Ames). This work was supported in part by NASA grant ATP02-0043-0056 and O5-BEFS-05-0044. JvM, MK, and MCM were also supported in part by the Research Associateship Programs Office of the National Research Council and Oak Ridge Associated Universities. 


\section{REFERENCES}

Baker, J., Centrella, J., Choi, D.-I., Koppitz, M., \& van Meter, J. (gr-qc/0511103)

Baker, J., Centrella, J., Choi, D.-I., Koppitz, M., \& van Meter, J. (gr-qc/0602026)

Bekenstein, J. D. 1973, ApJ, 183, 657

Blanchet, L., Qusailah, M. S. S., \& Will, C. M. 2005, ApJ, 635, 508

Boylan-Kolchin, M., Ma, C.-P., \& Quataert, E. 2004, ApJ, 613, L37

Brandt, S. \& Brügmann, B. 1997, Phys. Rev. Lett., 78, 3606

Bullock, J. S., et al. 2001, MNRAS, 321, 559

Campanelli, M. 2005, Class. Quant. Grav., 22, S387

Campanelli, M., Lousto, C. O., Marronetti, P., \& Zlochower, Y. (gr-qc/0511048)

Damour, T., \& Gopakumar, A. 2006, gr-qc/0602117

Favata, M., Hughes, S. A., \& Holz, D. E. 2004, ApJ, 607, L5

Fitchett, M. J. 1983, MNRAS, 203, 1049

Fitchett, M. J., \& Detweiler, S. 1984, MNRAS, 211, 933

Gültekin, K., Miller, M. C., \& Hamilton, D. P. 2004, ApJ, 616, 221

Gültekin, K., Miller, M. C., \& Hamilton, D. P. 2006, ApJ, 640, 156

Haiman, Z. 2004, ApJ, 613, 36

Herrman, F., Shoemaker, D., \& Laguna, P. 2006, gr-qc/0601026

Imbiriba, B., et al. 2004, Phys. Rev., D70, 124025

Kulkarni, S. R., Hut, P., \& McMillan, S. L. W. 1993, Nature, 364, 421

Libeskind, N. I., Cole, S., Frenk, C. S. \& Helly, J. C. 2005, MNRAS, submitted (astro$\mathrm{ph} / 0512073)$

MacNeice, P., Olson, K. M., Mobarry, C., deFainchtein, R. \& Packer 2000, Computer Physics Comm., 126, 330

Madau, P., \& Quataert, E. 2004, ApJ, 606, L17 
Merritt, D., Milosavljevic, M., Favata, M., Hughes, S. A., \& Holz, D. E. 2004, ApJ, 607, L9

Micic, M., Abel, T., \& Sigurdsson, S. 2005, MNRAS, submitted (astro-ph/0512123)

Miller, M. C., \& Colbert, E. J. M. 2004, IJMPD, 13, 1

Miller, M. C., \& Hamilton, D. P. 2002a, MNRAS, 330, 232

Miller, M. C., \& Hamilton, D. P. 2002b, ApJ, 576, 894

Mo, H. J., \& White, S. D. M. 2002, MNRAS, 336, 112

Mouri, H., \& Taniguchi, Y. 2002a, ApJ, 566, L17

Mouri, H., \& Taniguchi, Y. 2002b, ApJ, 580, 844

Navarro, J. F., Frenk, C. S., \& White, S. D. M. 1997, ApJ, 490, 493

O’Leary, R. M., Rasio, F. A., Fregeau, J. M., Ivanova, N., \& O’Shaughnessy, R. 2006, ApJ, 637,937

Peres, A. 1962, Phys. Rev., 128, 2471

Pretorius, F. 2005 Phys. Rev. Lett., 95, 121101

Pretorius, F. 2006 (gr-qc/0602115)

Redmount, I. H., \& Rees, M. J. 1989, Commun. Astrophys., 14, 165

Sigurdsson, S., \& Hernquist, L. 1993, Nature, 364, 423

Taffoni, G., Mayer, L., Colpi, M., \& Governato, F. 2003, ApJ, 341, 434

Taniguchi, Y., Shioya, Y., Tsuru, T. G., \& Ikeuchi, S. 2000, PASJ, 52, 533

Volonteri, M., \& Perna, R. 2005, MNRAS, 358, 913

Wiseman, A. G. 1992, PRD, 46, 1517

Yoo, J., \& Miralda-Escudé, J. 2004, ApJ, 614, L25 


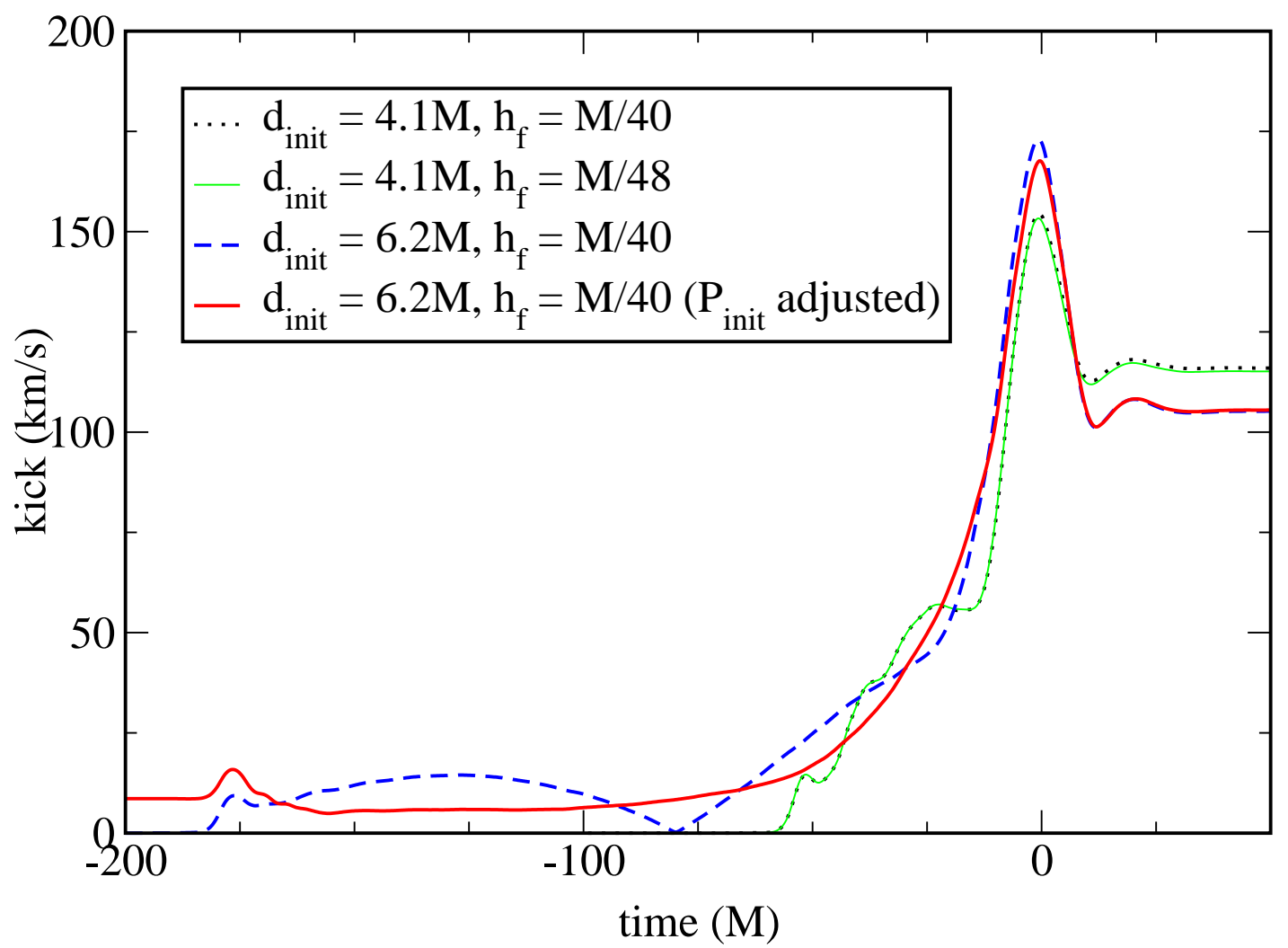

Fig. 1. - The magnitude of the radiated momentum, as a function of time, from three different simulations. For an initial separation between the black hole punctures of coordinate distance $d_{\text {init }}=4.1 M_{0}$, the final values of the momenta from two different resolutions are shown to agree to within $1 \%$. For an initial separation of coordinate distance $d_{\text {init }}=6.2 M_{0}$, the final value of the momentum is shown to agree with that of the closer initial separation to within $10 \%$. Also shown, the $d_{\text {init }}=6.2 M_{0}$ momentum is recomputed assuming a nonzero initial momentum vector $P_{\text {init }}$, estimated so as to give a more monotonically increasing curve and presumably thereby to approximate the momentum radiated up to this point by black holes of initially infinite coordinate separation. The final kick is nearly the same for all curves, strongly suggesting that the result is correct to better than $10 \%$. 


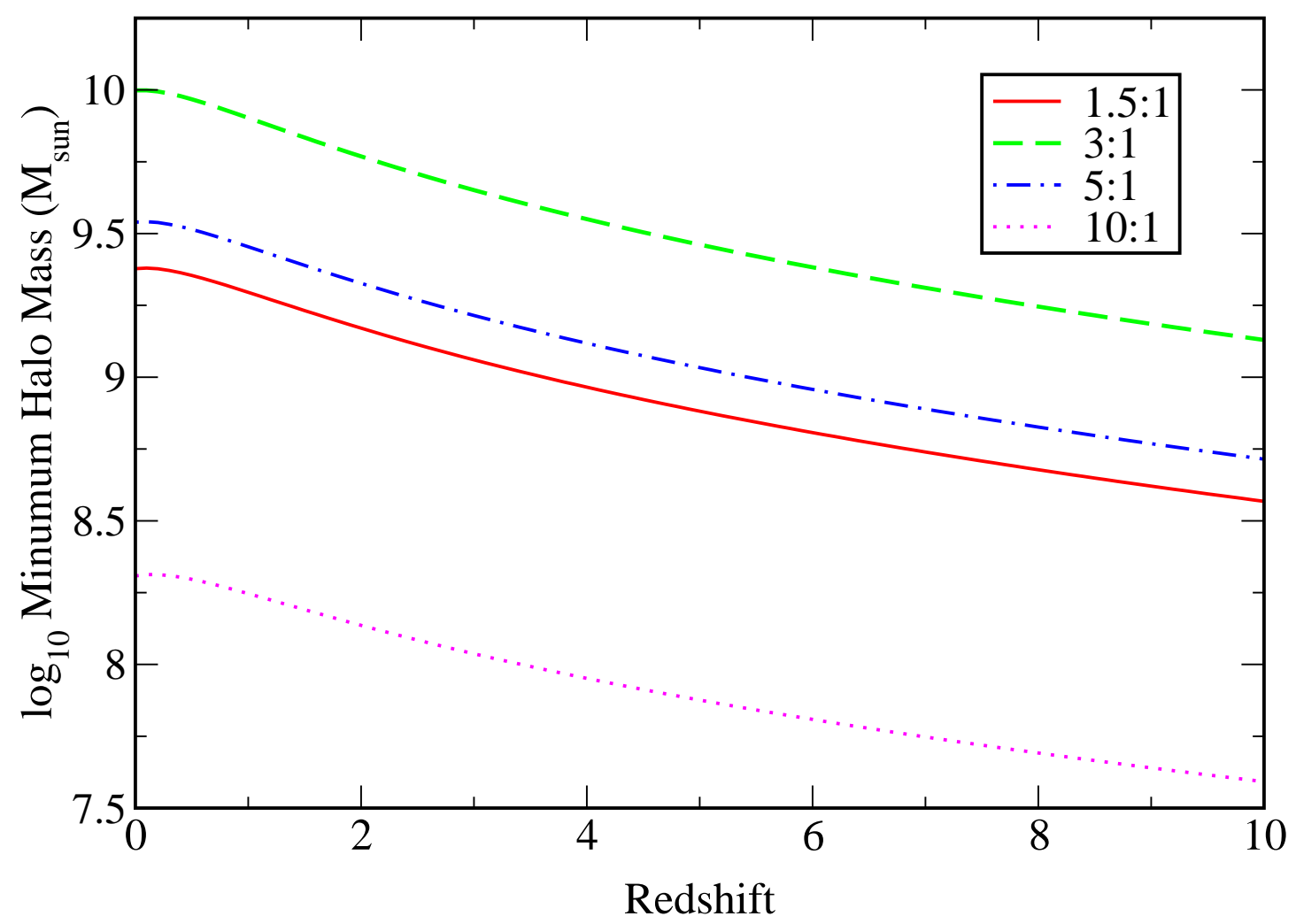

Fig. 2.- Minimum mass of a dark matter halo at a given redshift required to retain the product of the merger of two nonspinning black holes with a mass ratio indicated on the curve. Details of the computation are in the text; note that we use the Fitchett (1983) analytical estimate of the mass ratio dependence. This figure indicates that early halos might lose merger remnants because of the kick, but in the current universe only the smallest dwarf galaxies or globular clusters could have black holes ejected because of gravitational radiation recoil. 